\title{
ARTICLE
}

Received 14 Aug 2014 | Accepted 22 Jul 2015 | Published 25 Sep $2015 \quad$ DOl: 10.1038/ncomms9139

\section{An Arabidopsis PWI and RRM motif-containing protein is critical for pre-mRNA splicing and ABA responses}

Xiangqiang Zhan¹, Bilian Qian², Fengqiu Cao¹, Wenwu Wu1, Lan Yang¹, Qingmei Guan², Xianbin Gu², Pengcheng Wang ${ }^{3}$, Temiloluwa A. Okusolubo², Stephanie L. Dunn², Jian-Kang Zhu ${ }^{1,3}$ \& Jianhua Zhu ${ }^{2}$

The phytohormone abscisic acid ( $A B A)$ is important for growth, development and stress responses in plants. Recent research has identified $A B A$ receptors and signalling components that regulate seed germination and stomatal closure. However, proteins that regulate ABA signalling remain poorly understood. Here we use a forward-genetic screen to identify rbm25-1 and rbm25-2, two Arabidopsis mutants with increased sensitivity to growth inhibition by ABA. Using RNA-seq, we found that RBM25 controls the splicing of many pre-mRNAs. The protein phosphatase $2 \mathrm{CHAB1}$, a critical component in $\mathrm{ABA}$ signalling, shows a dramatic defect in pre-mRNA splicing in rbm25 mutants. Ectopic expression of a HAB1 complementary DNA derived from wild-type mRNAs partially suppresses the rbm25-2 mutant phenotype. We suggest that RNA splicing is of particular importance for plant response to ABA and that the splicing factor RBM25 has a critical role in this response.

\footnotetext{
${ }^{1}$ Shanghai Center for Plant Stress Biology, Shanghai Institutes of Biological Sciences, Chinese Academy of Sciences, Shanghai 200032, China. ${ }^{2}$ Department of Plant Science and Landscape Architecture, University of Maryland, 2121 Plant Sciences Building, College Park, Maryland 20742, USA. ${ }^{3}$ Department of Horticulture and Landscape Architecture, Purdue University, West Lafayette, Indiana 47907, USA. Correspondence and requests for materials should be addressed to X.Z. (email: xqzhan@sibs.ac.cn) or to J.Z. (email: jhzhu@umd.edu).
} 
T he phytohormone abscisic acid (ABA) is critical for many processes in the life cycle of plants, such as the promotion of seed maturation, establishment and maintenance of seed dormancy, regulation of stomatal aperture and adaptation to environmental stresses $^{1-3}$. On exposure to environmental stresses, plants rapidly increase their $A B A$ content as a result of increased $\mathrm{ABA}$ synthesis, decreased $\mathrm{ABA}$ catabolism, decreased formation of inactive $\mathrm{ABA}$ conjugates, or a combination of these factors ${ }^{4}$. The de novo biosynthesis of $\mathrm{ABA}$ is through the cleavage of a $\mathrm{C}_{40}$ carotenoid precursor, followed by the conversion of the intermediate xanthoxin to $A B A$ via $A B A$ aldehyde $^{2}$. The cleavage step catalysed by a 9-cis epoxycarotenoid dioxygenase (NCED) has generally been considered the rate-limiting step in the pathway ${ }^{4,5}$. Wang et al. ${ }^{6}$ found that $C E D 1$ (for NCED defective 1 ), which is a putative $\alpha / \beta$ hydrolase domain-containing protein and is allelic to the BODYGUARD gene that is essential for cuticle biogenesis, plays an important role in regulating the expression of NCED3 and genes encoding other signalling components downstream of $\mathrm{ABA}$ biosynthesis.

Following ABA biosynthesis, multiple signal transduction pathways, which amplify the primary signal produced when $\mathrm{ABA}$ binds to its receptors, are required to control the ABAregulated adaptive responses. These adaptive responses include stomatal closure (which allows the plant to conserve water), the accumulation of compatible osmolytes such as proline ${ }^{7}$, and the differential expression of a wide array of stress-responsive genes $^{8,9}$. The core ABA signal transduction pathway is mediated by receptors in the PYR/PYL/RCAR family, which, on binding to $\mathrm{ABA}$, inhibit the type $2 \mathrm{C}$ protein phosphatases (PP2Cs) such as ABI1, ABI2 and HAB1 (refs 3,10-12). The PP2Cs are negative regulators of the ABA signalling pathway ${ }^{13,14}$. Inhibition of the PP2Cs leads to the activation of protein kinases in the sucrose non-fermenting related kinase 2 (SnRK2) family. In the absence of ABA, the PP2Cs can directly inactivate SnRK2 kinases by dephosphorylating a critical amino acid residue in their kinase activation loop ${ }^{15,16}$. These kinases are required for the subsequent regulation of the activities of downstream effectors in $\mathrm{ABA}$ signalling.

Since the three SnRK2 kinases, SnRK2.2, SnRK2.3 and SnRK2.6, control virtually all of the ABA responses in plants, the substrate proteins of the SnRK2s function as the downstream effectors of ABA responses ${ }^{17}$. The best known of the substrates include several transcription factors and ion channels ${ }^{18-21}$. The basic leucine zipper family transcription factors such as $\mathrm{ABI} 5$ and other $\mathrm{ABA}$ response factors or ABA-responsive element-binding factors (AREBs) control the transcription of ABA-responsive genes ${ }^{18,20}$. The ion channels such as the slow anion channel SLAC1 and the potassium channel KAT1 are critical for guard cell regulation by $\mathrm{ABA}^{19,21-24}$. Recent phosphoproteomics studies identified dozens of additional substrate proteins of the SnRK2s including several proteins with potential roles in RNA processing and proteins involved in chloroplast function ${ }^{25,26}$. These newly identified substrate proteins are potential effectors of ABA signalling, although their functions in $\mathrm{ABA}$ responses are largely unknown.

To identify cellular factors important for $A B A$ responses, particularly ABA responses in leaf development, we conducted a genetic screen in Arabidopsis thaliana for mutants with defective leaf greening response to $A B A$ treatment. We report here that roal (regulator of $A B A$ response) mutants are hypersensitive to $\mathrm{ABA}(\mathrm{HAB})$ as indicated by impaired leaf greening in response to ABA. The ROA1/RBM25 locus defines a splicing factor required for the splicing of transcripts of many genes including those involved in ABA signal transduction pathways. Our work has revealed a critical role of pre-mRNA splicing in $\mathrm{ABA}$ responses in plants.

\section{Results}

roal mutants are hypersensitive to $\mathrm{ABA}$. Identification of a large number of mutants defective in seed germination or guard cell movements has led to the discovery of genes involved in $\mathrm{ABA}$ biosynthesis and its downstream signalling events ${ }^{3}$. High levels of $\mathrm{ABA}$ inhibit plant growth and development and cause leaves to become pale or yellow ${ }^{27,28}$. The cellular factors controlling these processes are poorly understood. To identify critical regulators for $\mathrm{ABA}$ responses at the seedling growth stage, we screened an A. thaliana transfer DNA (T-DNA) insertion population with known integration sites (the SALK collection from $A B R C$ ) for mutants with increased sensitivity to exogenous $\mathrm{ABA}$ in the growth medium in terms of root growth and leaf yellowing. A similar screen was performed with an in-house generated T-DNA insertional population in a TOUCH 4 promoter:luciferase reporter line ${ }^{29}$ (pTCH4:LUC). These mutants were designated as roa. The roal-1 (SALK_064472 from the SALK collection) and roal-2 (from the pTCH4:LUC population) mutants were chosen for in-depth characterization (Supplementary Fig. 1a). To compare roal-1 and roa1-2 side-byside, roa 1-2 was backcrossed with Col-0 and roa1-2 allele without the pTCH4:LUC transgene was obtained. In the absence of exogenous ABA, roal-1 and roal-2 seedlings do not exhibit obvious growth defects (Fig. 1a). However, after being transferred to media supplemented with $5 \mu \mathrm{M} \mathrm{ABA}$, the newly emerged leaves in roal-1 and roal-2 seedlings are yellow, whereas all of the leaves in the wild-type seedlings are green (Fig. 1a). The mutants also had reduced primary root elongation in response to $5 \mu \mathrm{M} \mathrm{ABA}$ (Fig. 1a,b).There is substantially less accumulation of chlorophylls in ABA-treated roa1-1 and roa1-2 leaves (Fig. 1a,c). Genetic analysis showed that both roa1-1 and roa1-2 mutations are recessive and are caused by mutations in a single nuclear gene (Supplementary Data 1). Pair-wise crosses between roal-1 and roal-2 suggested that the roa1-1 and roal-2 mutations are allelic to each other (Fig. 1d).

To investigate whether the roa1-1 and roal-2 mutations alter plant responses to other plant hormones or abiotic stress, we examined the responses of roal-1 and roal-2 plants to heat stress and two additional hormones. Sensitivity of the soil-grown roa1-1 and roa1-2 plants at both vegetative and reproductive stages to heat stress is not altered when they are compared with the wildtype plants (Supplementary Fig. 1b). We used the precursor of ethylene biosynthesis, 1-amino-cyclopropane-1-carboxylic acid (ACC), to examine the effect of ethylene on hypocotyl elongation of dark grown roa1-1 and roa1-2 seedlings. Hypocotyl elongation of roa1-1, roa1-2 and wild-type seedlings was equally inhibited by exogenous application of ACC (Fig. 1e; Supplementary Fig. 1c). We further examined effects of auxin on hypocotyl and root elongation of roal-1 and roa1-2 seedlings. roa1-1 and roa1-2 seedlings essentially responded to the same extent to indole-3-acetic acid in the growth media (Fig. 1f; Supplementary Fig. 1d,e). Therefore, while ROA1 may be involved in the response to stresses and hormones other than $\mathrm{ABA}$, we find no evidence of its involvement in the response to auxin, ethylene or heat.

ROA1 encodes a PWI and RRM motif-containing protein. Although roa1-1/SALK_064472 was annotated as having a T-DNA insertion in the At1g60200 gene, we could not find any T-DNA insertion in this gene. We carried out thermal asymmetric interlaced PCR (TAIL-PCR) analysis and identified a T-DNA insertion downstream of At1g60200 in roa1-1 (Fig. 2a). Although roal-2 also came from a T-DNA mutant population, there is no T-DNA insertion in roal-2. To identify the gene responsible for the roal-1 and roal-2 mutant phenotypes, we prepared mapping populations by crossing roa1-1 and roa1-2 

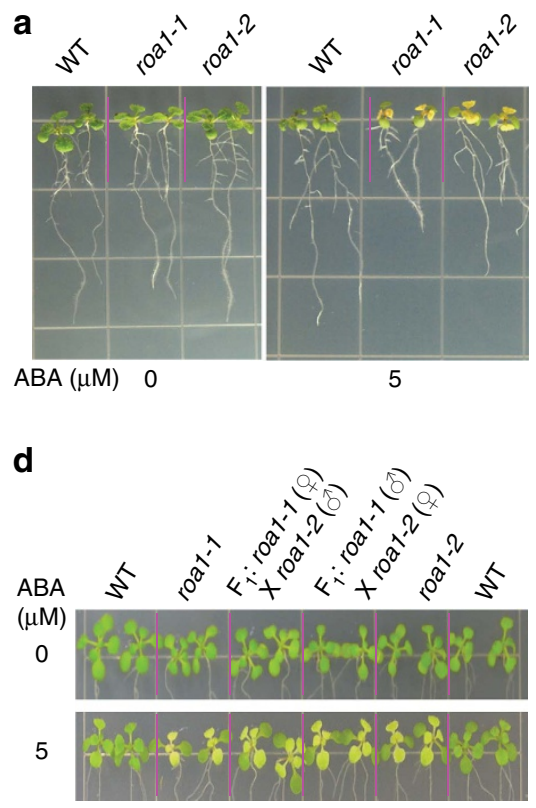

b
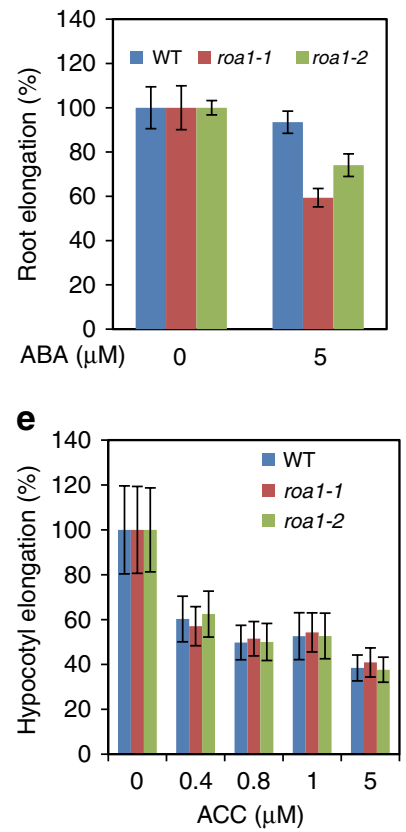

c
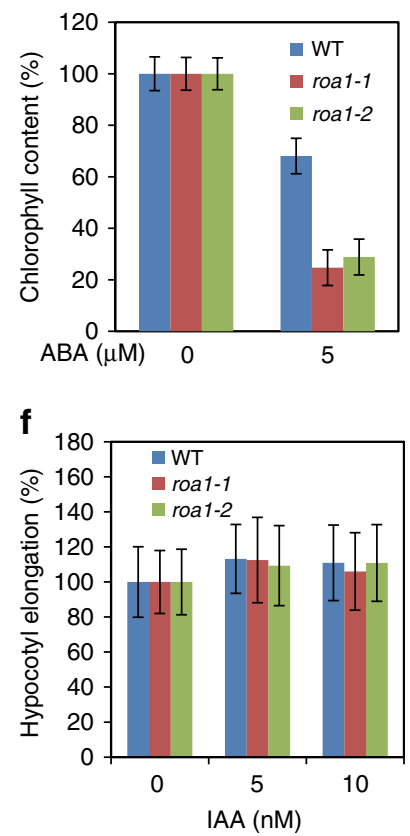

Figure 1 | Sensitivity of roa1 mutants to ABA, ethylene precursor ACC and auxin. Five-day-old seedlings grown in MS medium were transferred to MS

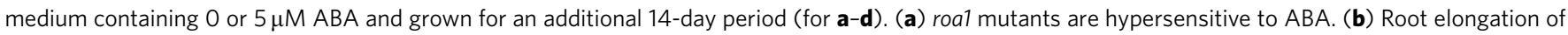

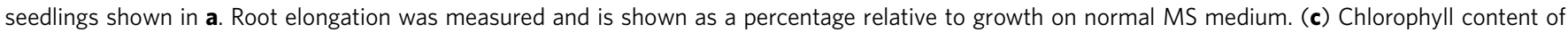
seedlings shown in $\mathbf{a}$. (d) Sensitivity of $\mathrm{F}_{1}$ seedlings from pair-wised crosses between roa1-1 and roa1-2 to ABA. Hypocotyl elongation of wild-type, roa1-1 and roa1-2 seedlings (for e-f). Seeds were germinated directly on medium containing various levels of ACC (in e; in darkness) or indole-3-acetic acid (in f; 8-h light $\left(80 \mu \mathrm{mol} \mathrm{m}-2 \mathrm{~s}^{-1}\right)$ and 16-h dark cycle). Seedlings were allowed to grow for an additional 3-day (in e) or 5-day period (in f) after germination. Values represent mean \pm s.d. ( $n=20$ in $\mathbf{b}, 8-10$ pools of $4-5$ seedlings in $\mathbf{c}, 49-135$ in e and $89-125$ in $\mathbf{f}$ ).

mutants with Landsberg erecta wild-type plants and performed map-based cloning for the ROA1 locus. Genetic mapping pinpointed $R O A 1$ to a genomic region that contains the At1g60200 gene. In the roa1-1 mutant, we identified a single nucleotide substitution in At1g60200, and this substitution changes alanine at position 899 to valine in the deduced polypeptide (Fig. 2a,b). The roa1-2 mutation also has a single nucleotide substitution in At1g60200, which results in the change of glutamine at position 570 to a premature stop codon in the deduced polypeptide (Fig. 2a,b). We confirmed the ROA1 gene is At1g60200 by functional complementation analysis. The wild-type At1g60200 gene driven by its native promoter complemented the roa1-1 and roa12 mutant phenotype (Fig. 2d,e). ROA1 expression is severely disrupted in roa1-1, possibly due to the T-DNA insertion downstream of ROA1 (Fig. 2f).

ROA1 encodes a putative PWI and RRM motif-containing protein with similarities to the human RNA-binding protein 25 (HsRBM25) (Fig. 2b,c). Hereafter, we refer to ROA1 as Arabidopsis RBM25 and refer to roa1-1 and roa1-2 mutant alleles as rbm25-1 and rbm25-2, respectively. Arabidopsis RBM25 shares substantial sequence similarity (50\% over the RRM motif and 71\% over the PWI motif) with the HsRBM25, which is known as a splicing factor ${ }^{30}$. Database searches revealed that RBM25 orthologues are present in other plant species including monocots (such as rice and maize) and dicots (such as soybean and tomato) (Fig. 2c; Supplementary Fig. 2). The last amino acid of RBM25 (changed to valine in rbm25-1) is conserved in nearly half of the plant RBM25 orthologues (Supplementary Fig. 2). As expected of a putative splicing factor, the RBM25-green fluorescent protein (GFP) fusion protein driven by the $R B M 25$ native promoter is localized in the nucleus of Arabidopsis root cells (Fig. 2g).
RBM25 negatively controls ABA responses in early development. Seed germination is sensitive to $\mathrm{ABA}$ and we were interested whether RBM25 plays a role in this process. $r b m 25-1$ is slightly hypersensitive to exogenous ABA at the stage of seed germination (Fig. 3a) and is substantially hypersensitive to ABA in post-germination seedling development (Fig. 3b,c). This hypersensitivity to $\mathrm{ABA}$ was suppressed in three independent complementation lines of rbm25-1 (Fig. 3a,b,c), indicating that loss-of-function of RBM25 results in this phenotype. ABA is also known to induce stomatal closure on drought stress. We performed water loss assays with detached shoots of soil-grown rbm 25 mutant plants to determine whether rbm 25 mutations alter plant transpirational water loss. Transpirational water loss rates are the same between the wild-type and $r b m 25$ mutants with or without exogenous application of ABA (Fig. 3d). These results suggest that rbm25 mutations do not alter stomatal responses to ABA.

The rbm25-1 mutation alters transcript accumulation profiles. We performed RNA-seq experiments to determine whether the rbm25-1 mutation affects transcript accumulation profiles and whether altered transcript accumulation profiles may help explain the increased sensitivity of rbm25-1 mutant to ABA treatment. We used the Illumina HiSeq 2500 System to sequence mRNA-seq libraries prepared from wild-type and rbm25-1 seedlings with three biological replicates and obtained a minimum of 14.7 million of pair-end clean sequence reads (Supplementary Data 2). Compared with those in the wild-type, 106 genes displayed higher transcript levels (by at least 2 -fold and with $P<0.05$ ), while 97 genes showed lower transcript levels in rbm25-1 under control conditions (Fig. 4a,b; Supplementary Data 2). The RNA-seq analysis also revealed that 185 genes displayed at least a 2 -fold 
a

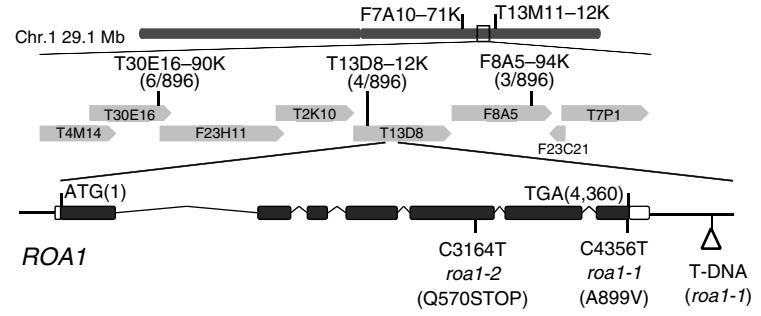

b

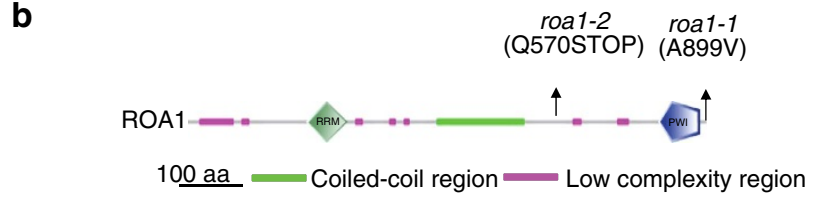

C

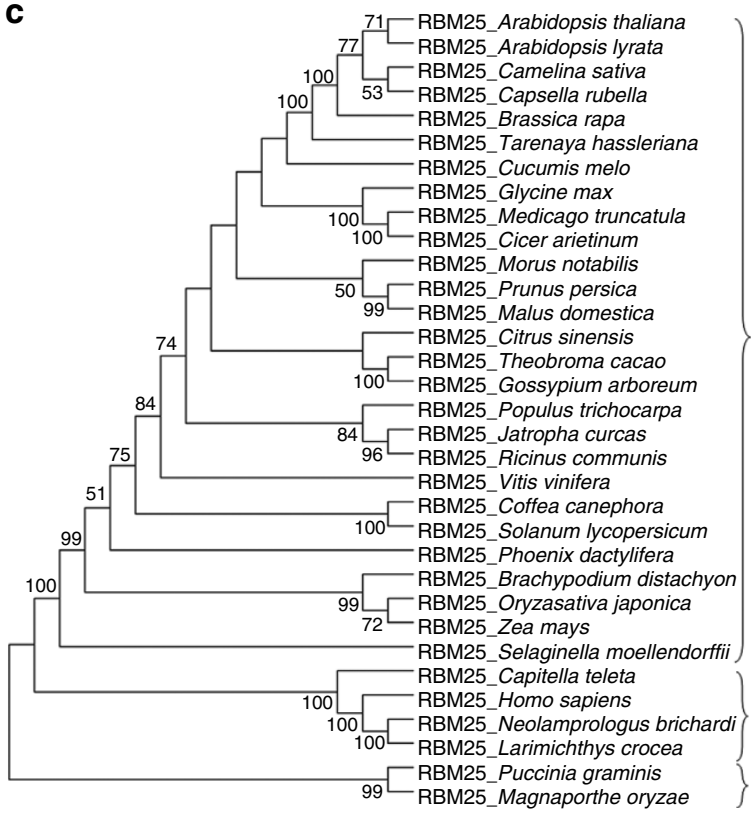

d

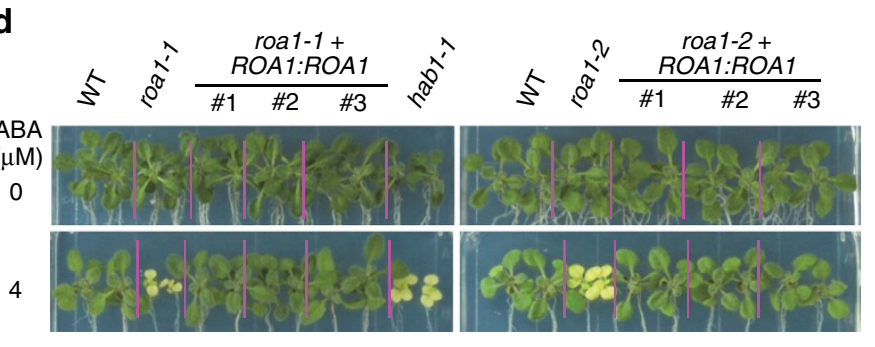

e

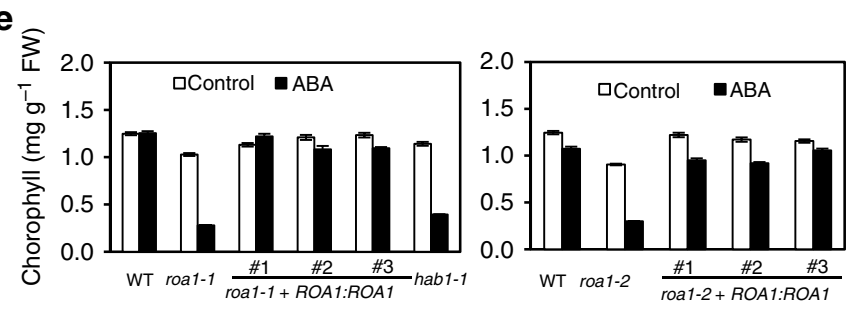

$\mathbf{f}$

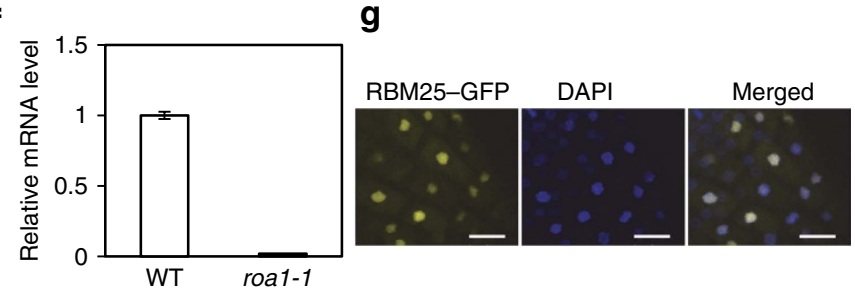

Figure 2 | Molecular cloning of ROA1. (a) Map-based cloning of ROA1. Structure of the ROA1 gene and positions of the roa1-1 and roa1-2 mutations are indicated. Filled boxes and empty boxes indicate exons and untranslated regions, respectively; the solid lines between boxes indicate introns. Positions are relative to translation start sites. (b) Structure of ROA1. Motifs are predicted by the SMART database. (c) Phylogenetic tree of RBM25 and its close orthologues from other organisms. The protein identities are the same as defined in Supplementary Fig. 2. The Bootstrap consensus tree was generated with MEGA6 (ref. 69). Protein sequences of RBM25 and its orthologues were aligned by MUSCLE and the Bootstrap consensus phylogenetic tree was reconstructed by the statistical method maximum likelihood using the Jones-Taylor-Thornton (JTT) model with a Bootstrap of 1000 . Cut-off value for consensus tree is 50\%. (d) Gene complementation analysis. (e) Chlorophyll content in plants shown in d. (f) Relative transcript levels of ROA7 in WT and roa1-1 plants as determined by qRT-PCR analysis. (g) Nuclear localization of the RBM25-GFP fusion protein. Scale bars, $2.5 \mu \mathrm{m}$. Values represent mean \pm s.d. ( $n=8$ in $\mathbf{e} ; 4$ in $\mathbf{f})$.

increase in transcript levels (relative to the wild type and with $P<0.05$ ), while 267 genes showed at least a 2-fold decrease in transcripts levels under $100 \mu \mathrm{M}$ ABA treatment for $6 \mathrm{~h}$ (Fig. 4a,b; Supplementary Data 2). Furthermore, we found that there are 37 genes whose transcript levels are increased in rbm25-1 plants under control and ABA treatment conditions (Fig. 4a; Supplementary Data 2). Similarly, there are 37 genes whose transcript levels are reduced in rbm25-1 plants under control and ABA treatment conditions (Fig. 4a; Supplementary Data 2). The differentially expressed genes in rbm25-1 encode proteins with diverse functions, and a substantial number of these proteins have predicted functions in stress responses (Fig. 4c; Supplementary Data 2). For example, under ABA treatment, 62 genes with annotated functions associated with response to abiotic or biotic stimulus or response to stress showed increased transcript levels in rbm25-1 mutant seedlings (Supplementary Data 2). This observation of large and preferential change in the expression of stress-related genes is consistent with the obviously more 'stressed' appearance of the mutant plants under ABA treatment (Figs $1 \mathrm{a}$ and $3 \mathrm{~b}$ ) as a result of their impaired ability to cope with $\mathrm{ABA}$ treatment. With quantitative reverse
transcription-PCR (qRT-PCR) analysis, we confirmed that the expression of At5g65080 and At $1 g 53490$ is substantially reduced in rbm 25 mutants, while expression of At4g33720 is increased in rbm25 mutants (Fig. 4d). At5g65080 encodes a MADS-box transcription factor. At1g53490 encodes a RING/U-box superfamily protein. At4g33720 encodes a CAP (cysteine-rich secretory proteins, Antigen 5 and Pathogenesis-related 1 protein) superfamily protein.

RBM25 regulates the splicing of pre-mRNAs. RBM25 has similar functional domains as the human splicing factor HsRBM25. To determine whether RBM25 functions in pre-mRNA splicing, we examined our RNA-seq data sets to look for potential effects of rbm25-1 mutation on pre-mRNA splicing. The analysis revealed that 359 genes have splicing defects in untreated rbm25-1, while 416 genes show splicing defects in ABA-treated rbm25-1 (Fig. 5a; Supplementary Data 3). Our RNA-seq data analysis further revealed that the rbm25-1 mutation causes defects in splicing of gene transcripts in eight different alternative splicing categories: alternative acceptor, alternative donor, cassette exon, intron retention (IR), coordinate 
a

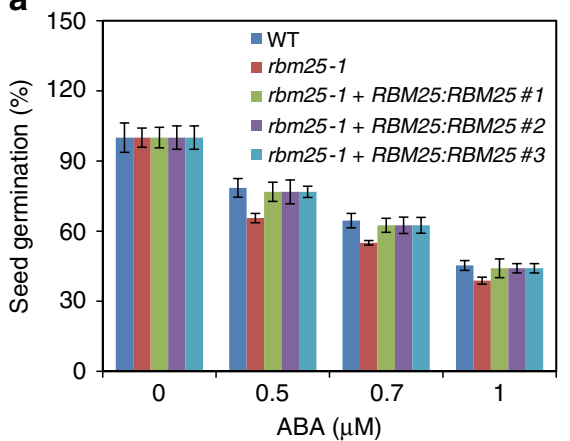

C

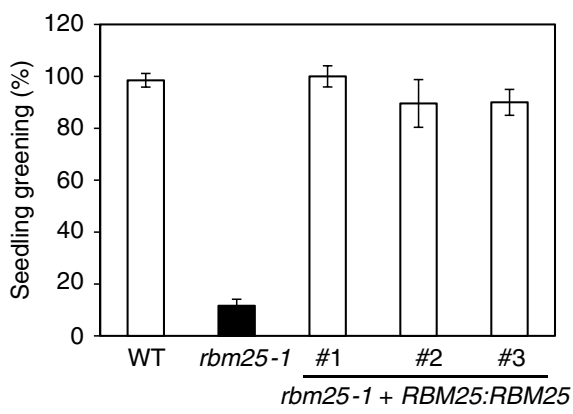

b

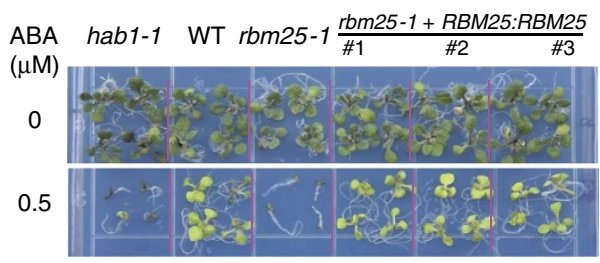

d

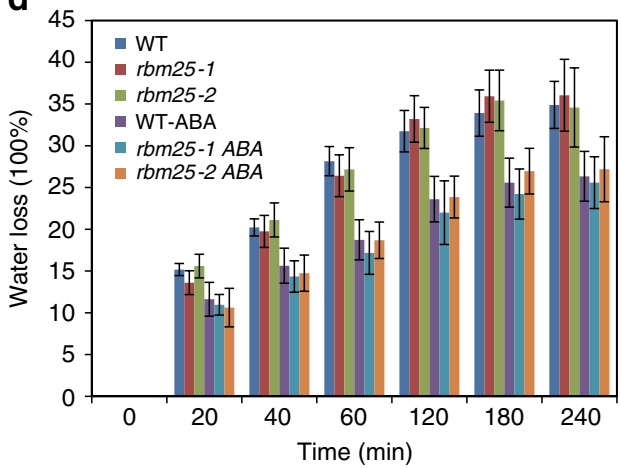

Figure 3 | The rbm25-1 mutation alters sensitivity to ABA in seed germination and post-germination seedling development, but does not affect water loss rates. (a) Seed germination of WT, rbm25-1 and rbm25-1 complementation lines. Seeds (50-60 per genotype) were sown on surface of filter paper soaked with various levels of ABA. Seeds were considered germinated when radicles penetrate the seed coats. The percentage of seeds that had germinated 6 days after sowing was determined. (b) Post-germination seedling development of WT, rbm25-1 and rbm25-1 complementation lines subjected to 0 or $0.5 \mu \mathrm{M}$ ABA. Photograph was taken 16 days after sowing seeds. (c) The percentage of green seedlings in $\mathbf{b}$. Seedlings with fully developed green cotyledons and at least one true green leaf were considered green. (d) Shoots from rbm25-1, rbm25-2 and wild-type plants at the rosette stage were placed on weighing trays and allowed to dry slowly at constant temperature $\left(25^{\circ} \mathrm{C}\right)$ and humidity $(\sim 50 \%)$. Weights of shoots were determined over a 4 -h period. $\mathrm{ABA}, 100 \mu \mathrm{M} \mathrm{ABA}$ for $3 \mathrm{~h}$. Values are mean \pm s.d. ( $n=8$ in $\mathbf{a}, 4$ (number of MS medium plates containing 0 or $0.5 \mu \mathrm{M}$ ABA; each plate has $30-40$ seedlings per genotype in $\mathbf{b}, 8$ in $\mathbf{d}$ ).

cassette exon, alternative first exon, alternative last exon and mutually exclusive exon (Fig. 5b; Supplementary Data 3). We designed primers unique to the intron of genes that are retained in the rbm25-1 plants and carried out semi-qRT-PCR assays to validate the IR results from the RNA-seq analysis. For RT-PCR analysis, we selected At4g16143, At5g09330, At2g42010, At3g53340, At4g32040 and At4g35800. At4g16143 encodes importin alpha isoform 2. At5g09330 encodes NAC domaincontaining protein 82. At4g42010 encodes phospholipase D beta 1. At3g53340 encodes nuclear factor Y, subunit B10. At4g32040 encodes KNOTTED1-like homeobox gene 5. At4g35800 encodes the unique largest subunit of nuclear DNA-dependent RNA II. The RT-PCR assays found that transcripts of At4g16143, At5g09330, At2g42010, At3g53340, At4g32040 and At4g35800 contain an intron in rbm25-1 and rbm25-2 with or without ABA treatment (Fig. 5c,e; Supplementary Fig. 3). Thus, the RT-PCR results confirmed the RNA-seq data of IR events in rbm25-1. RT-PCR analysis confirmed that exon 4 of At $2 g 48120$ is skipped in rbm 25 mutants under both control and ABA treatment conditions (Fig. 5c; Supplementary Figs 3 and 4). At $2 g 48120$ encodes the pale cress protein involved in chloroplast mRNA maturation ${ }^{31}$.

The genes carrying defective alternative splicing patterns in rbm25-1 plants under control or ABA treatment conditions encode proteins with diverse functions in many biological processes, and the predicted roles of a good portion of these genes involve responses to abiotic or biotic stresses (Supplementary Data 3). It is possible that altered activities of mis-spliced genes in unstressed rbm25-1 mutant account for differentially expressed genes in this mutant under control conditions as determined by RNA-seq analyses. There are 31 genes with a predicted role in DNA-dependent transcription in each category of abnormal splicing in unstressed rbm25-1 mutant (Supplementary Data 3). These genes include promoter-specific transcription factors, general transcription factors and RNA polymerase II subunits.

We reasoned that disrupted function of the mis-spliced genes in rbm25-1 plants should at least partly explain the impaired responses of rbm25 mutants to $\mathrm{ABA}$ treatment including alterations in gene expression and increased sensitivity of rbm 25 mutants to ABA in terms of root growth and leaf yellowing. Thirty genes involved in transcription were found to have splicing defects in their transcripts in rbm25-1 mutant under ABA treatment (Supplementary Data 3). These genes include the largest subunit of DNA-dependent RNA polymerase II, TATA-binding proteins, a nuclear factor, promoter-specific transcription factors and the subunits of the mediator complex. The mediators are involved in regulation of transcription from RNA polymerase $\mathrm{II}^{32}$. Loss-of-function or reduced activities of these genes will certainly lead to altered expression of genes in rbm25-1 mutant under ABA treatment. Although genes carrying splicing defects in rbm25-1 mutant under ABA treatment encode proteins involved in diverse biological processes, a substantial portion of the genes encode proteins that are predicted to function in biotic or abiotic stress-response pathways (Supplementary Data 3). In addition, the calcium- 


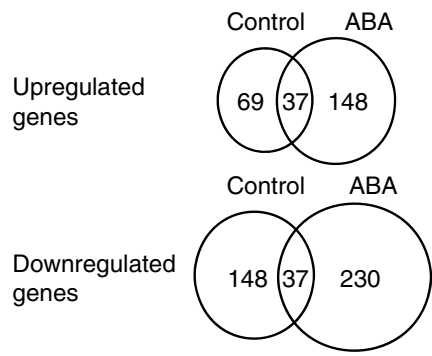

b

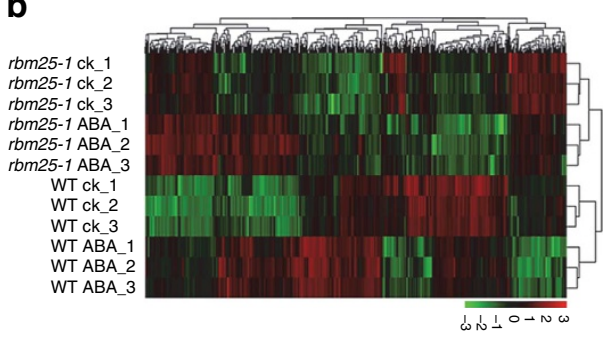

c

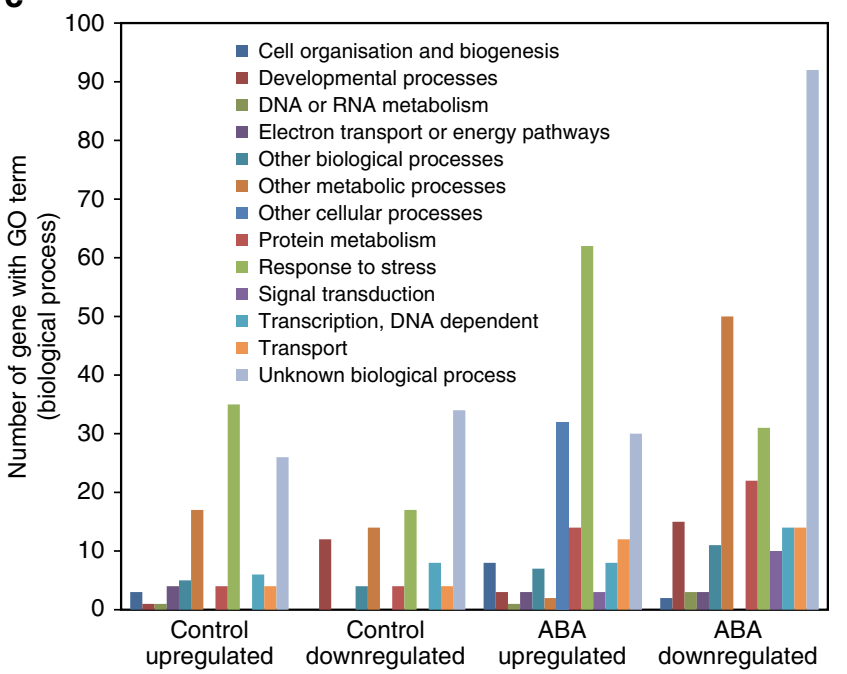

d

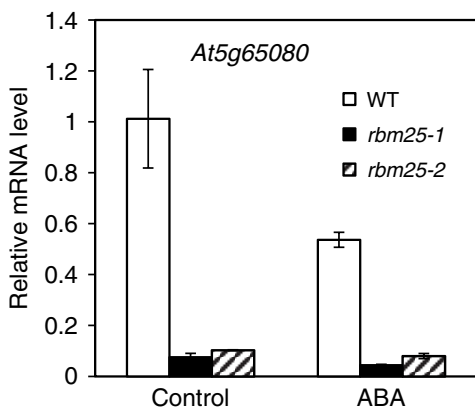

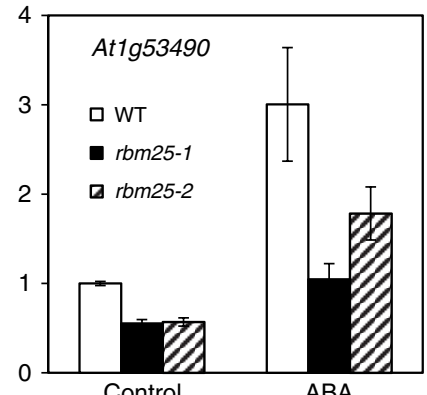

Control

ABA

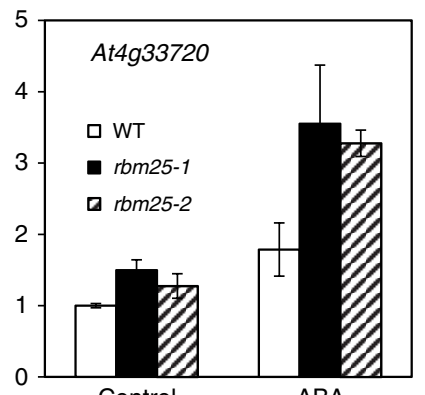

Figure 4 | The rbm25-1 mutation causes disrupted gene expression as determined in RNA-seq experiments. (a) Summary of differentially expressed genes in rbm25-1. (b) Heat map of differentially expressed genes in rbm25-1. Samples (rows) and genes (column) are hierarchically clustered via Pearson correlation. (c) GO term distribution of differentially expressed genes in rbm25-1. (d) Validation of differential gene expression portion of the RNA-sea results by qRT-PCR analysis. Values represent mean \pm s.d. $(n=3)$.

sensing receptor and $\mathrm{HAB1}$ are known regulators of $\mathrm{ABA}$ responses ${ }^{33-35}$. There are additional PP2Cs whose transcripts are mis-spliced in ABA-treated rbm25-1 mutant. Like HAB1, these PP2Cs may function as negative regulators of ABA signalling. Finally, impaired functions of four splicing factors (SC35-like splicing factor 33 encoded by At1g55310, CC1-like splicing factor encoded by At2g16940, U2 snRNP splicing factor encoded by At1g60900 and splicing endonuclease 1 encoded by At3g45590) may contribute to defects of alternative splicing of gene transcripts in rbm25-1.

$\mathrm{ABA}$ represses the expression of genes involved in photosynthesis including the small subunit of ribulose-1,5bisphosphate carboxylase/oxygenase (Rubisco) ( $r b c S$ genes) and chlorophyll a/b-binding proteins $\mathrm{s}^{36,37}$. Related to this aspect of cellular function, we found mis-splicing of a group of genes that are involved in biogenesis and functionality of chloroplasts in ABA-treated rbm25-1 mutant. These genes include pale cress protein (encoded by At2g48120; the mutant of this gene has reduced chlorophyll content ${ }^{31}$ ), the PsbQ subunit of the oxygenevolving complex of photosystem II (encoded by At4g21280) ${ }^{38}$, starch branching enzyme 2.2 (encoded by At5g03650; it is transcriptionally regulated by the ABA-insensitive $\left.4(\mathrm{ABI} 4)^{39}\right)$, UDP-glycosyltransferase superfamily protein involved in flavonoid biosynthetic process (encoded by At4g09500) and plastid transcriptionally active 5 (PTAC5, encoded by At4g13670 (ref. 40)) (Supplementary Data 3). Mis-splicing for the transcripts of these proteins in rbm25-1 mutant may be associated or even contribute to the leaf yellowing phenotype of the mutant. Finally, we observed that a cyclin-dependent protein kinase (CDKC2 encoded by At5g64960) is mis-spliced in rbm25-1 under ABA treatment (Supplementary Data 3). CDKC2 colocalizes with spliceosomal components in a manner dependent on the transcriptional status of the cells and on CDKC2-kinase activity. Expression of $\mathrm{CDKC} 2$ modifies the location of spliceosomal components ${ }^{41}$. Thus, disruption of CDKC2 may exacerbate the splicing defects caused by the rbm25-1 mutation.

RBM25 controls the alternative splicing of $H A B 1$ transcripts. Our RNA-seq analysis revealed that the last intron of At1g72770 (which encodes HAB1) is retained in rbm25-1 plants (Supplementary Data 3). HAB1 is one of the clade A PP2Cs that are co-receptors of $\mathrm{ABA}$ and important negative regulators of ABA signalling $14,33,42,43$. HAB1 transcripts are known to exist in three alternatively spliced forms (The Arabidopsis Information Resource, http://www.arabidopsis.org). In our efforts to confirm intron-retention event in rbm25-1 and rbm25-2 with RT-PCR and $\mathrm{qRT}-\mathrm{PCR}$ analyses, we found additional splicing defects of HAB1 in the rbm 25 mutants. The rbm 25 mutations cause altered accumulation of four alternatively spliced transcripts of $H A B 1$ under control and ABA treatment conditions (Fig. 6a,b). Because $H A B 1$ is upregulated by $A B A$, the defects of $H A B 1$ are more 
a

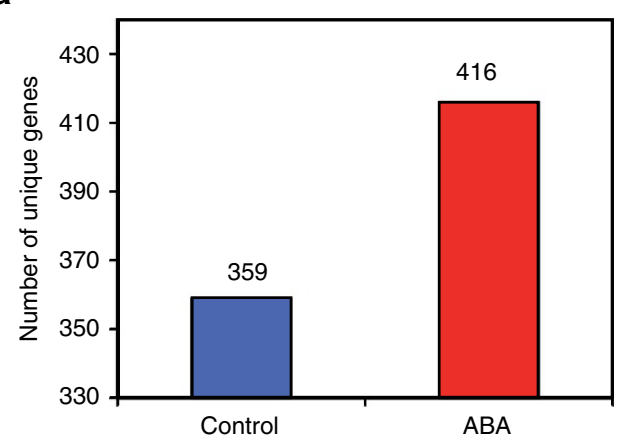

C

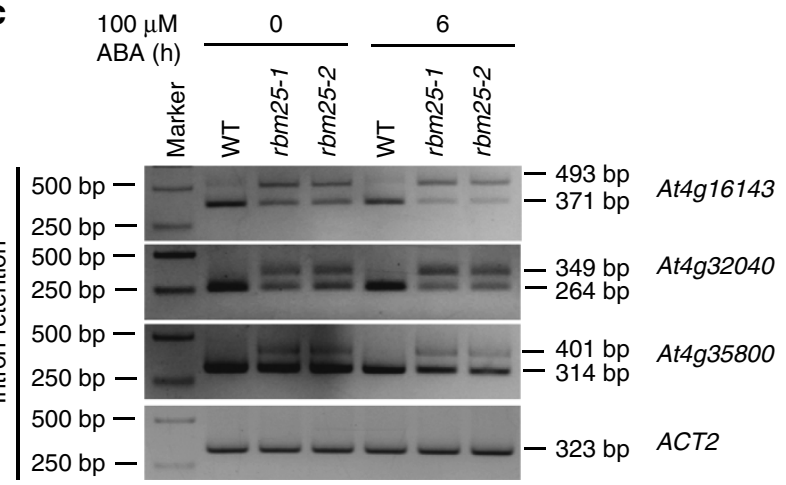

b

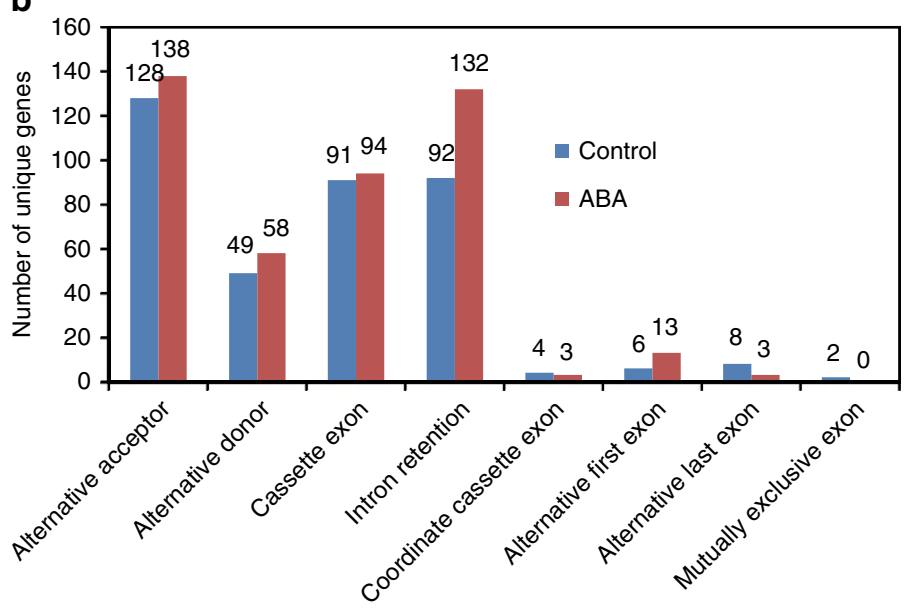

d

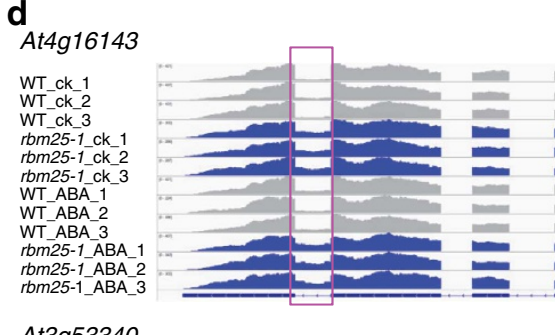

At3g53340

WT_ck_1
WT_ck_2
WT_ck_3

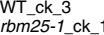

rbm25-1 ck-2
rbm25-1 ck- 23

WTABA-1

WT $A B A-2$

WT-ABA-3

rbm25-1_ABA-1
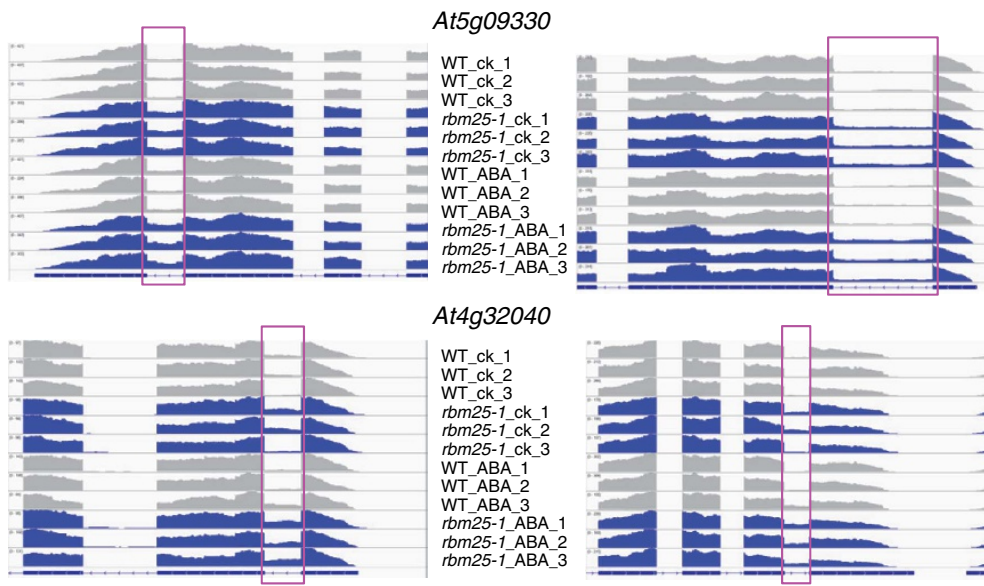

$100 \mu \mathrm{M}$

$\mathrm{ABA}(\mathrm{h})$

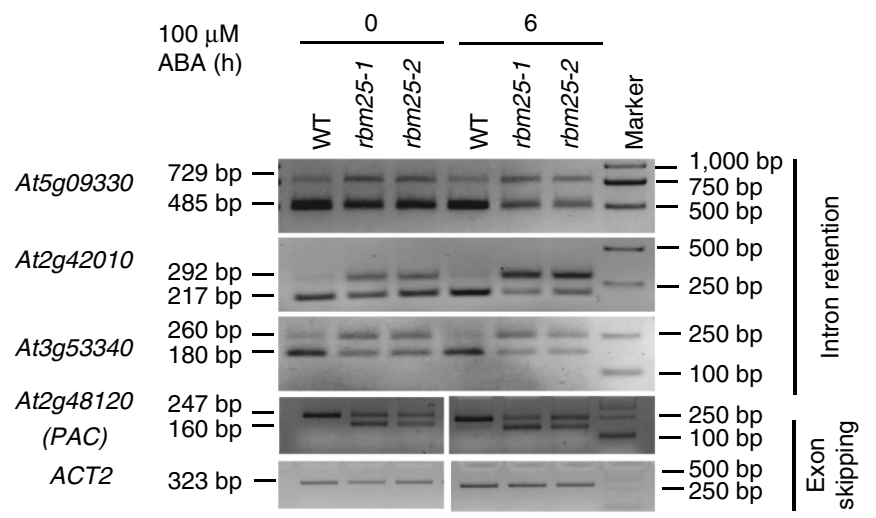

rbm25-1_ABA

At4g32040

WT-ck-1

WT_ck_2

rbm25-1_ck_1

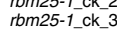

WT_ABA-1

WT_ABA_2

rbm25-1_ABA-1

rbm25-1_ABA_3

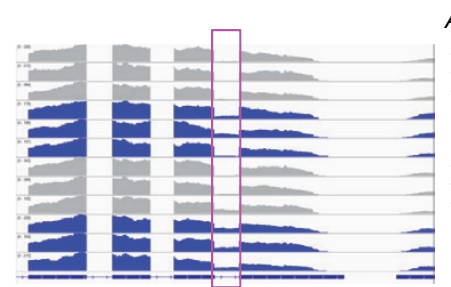

At2g42010

WT_ck 1

WT-ck-2

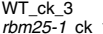

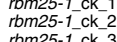

WT ABA -

WT-ABA-2

WT_ABA- 3

rbm25-1_ABA-1

rbm25-1-ABA 3
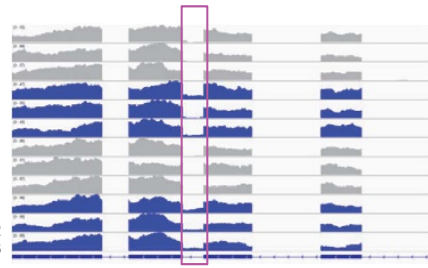

At4g35800

WT_ck_1

WT-ck-2
WT
WK- 3

rbm25-1 ck-1

rbm25-1 ck_2

WT ABA_-1

WT ABA-2

rbm25-1_ABA-1

rbm25-1_ABA-
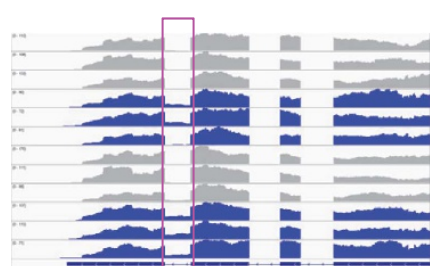

Figure 5 | The rbm25-1 mutation causes defects in alternative splicing of gene transcripts. (a) Summary of genes whose transcripts were abnormally spliced in rbm25-1 as determined by RNA-seq experiments. (b) Genes with defects in different types of alternative splicing patterns in rbm25-1 as determined by RNA-seq experiments. (c) Validation of intron-retention and exon-skipping events in WT, rbm25-1 and rbm25-2 plants as determined by RT-PCR analysis. (d) Visualization with the integrative genomic viewer (IGV) of intron-retention events in WT and rbm25-1 mutant plants. Annotated gene structures are shown (bottom), with thick lines representing exons and thin lines representing introns. Wiggle plots represent the normalized read coverage on an autoscale.

pronounced in these two mutants under ABA treatment. In rbm25-1 and rbm25-2 plants, HAB1.1 (At1g72770.1) displayed reduced expression level, while HAB1.2 (At1g72770.2) and HAB1.4 (At1g72770.4, a previously unannotated splicing variant) showed increased expression level (Fig. 6a,b). The HAB1.2 and HAB1.4 isoforms are the intron-retained $H A B 1$ transcripts identified in our RNA-seq experiments
(Supplementary Data 3). The expression level of HAB1.3 (At1g72770.3) is very low and there is no difference between the wild type and rbm 25 mutants for the accumulation of $H A B 1.3$ transcripts (Fig. 6b). Transcripts of HAB1.2 and HAB1.4 will likely produce non-functional, truncated polypeptides of HAB1 (Fig. 6c). Therefore, the overall manifestation of the defect in $H A B 1$ transcripts in $r b m 25-1$ and $r b m 25-2$ is the substantial 
a
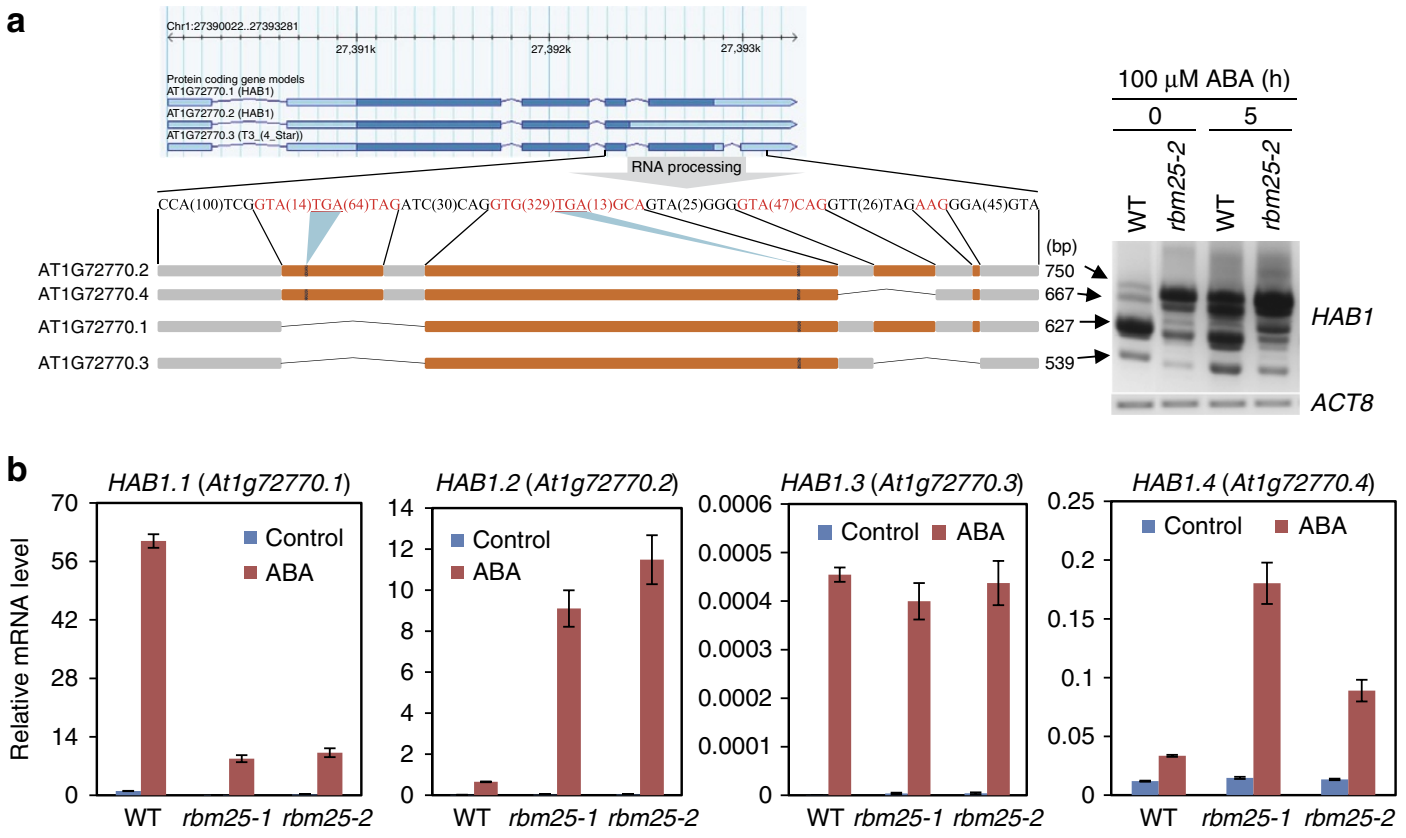

C

HAB1.1 nt 1201 GGT GAC AGA TAT CTG AAG CCA TAT GTG ATC CCA GAA 1236

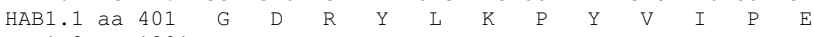

HAB1.2 nt 1201 GGT AAG CAT TGC TTC TTT TGA

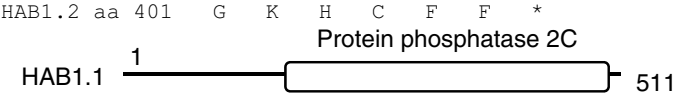

$\mathrm{HAB} 1.2 \stackrel{1}{\longrightarrow} 406$

d

$\begin{array}{lllll}\text { WT } & \text { rbm25-2 } & \begin{array}{l}\text { rbm25-2+ } \\ \text { 35S:HAB1.1 \#3 }\end{array} \text { 35S:HAB1.1 \#4 }\end{array}$ WT rbm25-2 $\begin{array}{ll}r b m 25-2+ & \text { rbm25-2+ } \\ 35 S: H A B 1.2 \# 3 & \text { 35S:HAB1.2 \#4 }\end{array}$
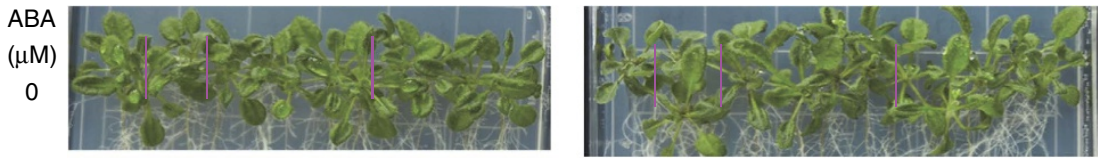

25

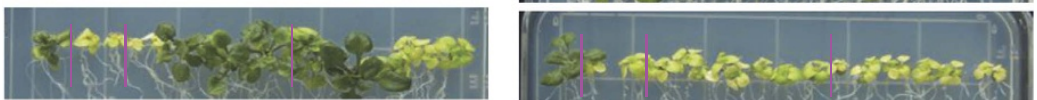

Figure 6 | RBM25 controls alternative splicing of HAB1. (a) Different alternative splicing variants of HAB1 in WT and rbm25-2 plants as determined by RT-PCR analysis followed by sequence analyses of cloned PCR products. ACT8 was used as the loading control. In the close-up view of the portion corresponding to most parts of the last two exons of At 1972770.1 , grey-shaded and orange-shaded boxes represent sequences in an exon, and folded lines between two boxes indicate introns in gene models of At1g72770.1, At7g72770.3 and At1g72770.4. (b) Relative transcript levels of different alternative splicing variants of $H A B 1$ (expression level of At1g72770.1 in unstressed WT was set to 1) in WT, rbm25-1 and rbm25-2 plants subjected to $100 \mu$ M ABA for 0 or $6 \mathrm{~h}$ as determined by qRT-PCR analysis. Values are mean \pm s.d. $(n=4)$. (c) Deduced C-terminal portion of HAB1.1 and HAB1.2. The predicted domain structures of HAB1.1 and HAB1.2 (HAB1.4 has the same structure as HAB1.2) are also presented. (d) Effect of HAB1 on ABA sensitivity of rbm25-1 mutant plants. Five-day-old seedlings grown in MS medium were transferred to MS medium containing 0 or $25 \mu \mathrm{M} \mathrm{ABA}$ and grown for an additional 20-day period.

reduction of $H A B 1.1$, the major transcript of $H A B 1$ in wild-type plants (Fig. 6b,c).

Ectopic expression of $H A B 1$ suppresses rbm25-1. Loss-offunction mutations in $H A B 1$ cause ABA hypersensitivity in early seedling growth and development ${ }^{14,33,44}$. Consistent with previous reports, the hab1-1 mutant is hypersensitive to ABA during seed germination and early seedling development (Fig. 3b). We observed that in response to ABA treatment hab1-1 mutant seedlings exhibit a leaf yellowing phenotype similar to that of $r b m 25$ mutants, although the yellowing is not as severe as in rbm25 (Fig. 2d). Considering the central role of HAB1 in ABA signalling and the seedling growth and development phenotypes of hab1-1 mutant plants in response to ABA treatment, we hypothesized that the defects in $H A B 1$ pre-mRNA splicing, particularly the dramatic reduction in $H A B 1.1$, may partially explain the leaf yellowing phenotype of ABA-treated rbm 25 mutant plants. To test this hypothesis, we ectopically expressed full-length $H A B 1.1$ complementary DNA (cDNA) under the CaMV 35S promoter in rbm25-2 mutant plants. As a control, we also expressed $H A B 1.2 \mathrm{cDNA}$ under the CaMV 35S promoter in rbm25-2 mutant plants. Two independent lines of $\mathrm{T}_{2}$ transgenic rbm25-2 seedlings were tested on media supplemented with $25 \mu \mathrm{M}$ ABA. The results show that the ectopic expression of $H A B 1.1$ but not $H A B 1.2$ could partially rescue the leaf yellowing phenotype of $r b m 25-2$ in response to $\mathrm{ABA}$ treatment (Fig. $6 \mathrm{~d}$ ). These results indicated that the $H A B 1.1$ but not the $H A B 1.2$ spliced form is functional in controlling leaf development under ABA treatment and the 
reduction of the $H A B 1.1$ form contributes significantly to the leaf yellowing phenotype of rbm 25 mutant plants.

ABA affects splicing of transcripts. We also examined the effect of $\mathrm{ABA}$ on the splicing pattern of transcripts in wild-type plants. $\mathrm{ABA}$ induces alternative splicing events in the transcripts of 27 genes in wild-type plants (Supplementary Data 3). Among these alternatively spliced genes in ABA-treated wild-type plants, two of them (At3g28670 and At3g24170) showed more severe splicing defects in ABA-treated rbm25-1 mutant. These results show that the ABA treatment causes defects in alternative splicing of premRNAs in wild-type plants, and the splicing defects become much more severe when the splicing factor RBM25 is impaired.

ABA regulates $R B M 25$ transcript level and post-translationally. $R O A 1$ expression is upregulated by ABA treatment (Fig. 7a). This is consistent with publicly available gene expression profiling data on ROA1 under ABA treatment obtained using whole genome tiling arrays ${ }^{45}$ (Supplementary Fig. 5). This is also consistent with the ABA-induced mutant phenotypes and more severe splicing defects in rbm 25 mutants under ABA treatment (Supplementary Data 3), and suggests a need for more RBM25 to strengthen premRNA splicing under ABA treatment.
Protein phosphorylation is central to ABA signalling ${ }^{18,20,23}$. $\mathrm{ABA}$ increases the phosphorylation levels of several splicing factors including arginine/serine-rich splicing factor 41, a PWI domain-containing protein, splicing factor U2af small subunit $A$ and serine/arginine-rich protein splicing factor RSZ32, while ABA also represses the phosphorylation levels of several splicing factors ${ }^{26}$. Because $R B M 25$ is upregulated by $\mathrm{ABA}$, we examined whether phosphorylation status of RBM25 may also be regulated by ABA. We used a novel mass-spectrometry-based label-free quantitation method that facilitates systematic profiling of plant phosphoproteome changes with high efficiency and accuracy ${ }^{46}$. This method employs synthetic peptide libraries tailored specifically as internal standards for complex phosphopeptide samples and accordingly, a local normalization algorithm, LAXIC, which calculates phosphopeptide abundance normalized locally with co-eluting library peptides. Our mass-spectrometry studies revealed that RBM25 is phosphorylated and ABA represses the phosphorylation level of RBM25 (Fig. 7b,c). Based on this data, we suggest that dephosphorylation of RBM25 may be important for its splicing function under ABA treatment. Alternatively, dephosphorylation of RBM25 may be important for its protein stability because phosphorylation often leads to protein degradation through ubiquitin-mediated proteolysis ${ }^{47,48}$.
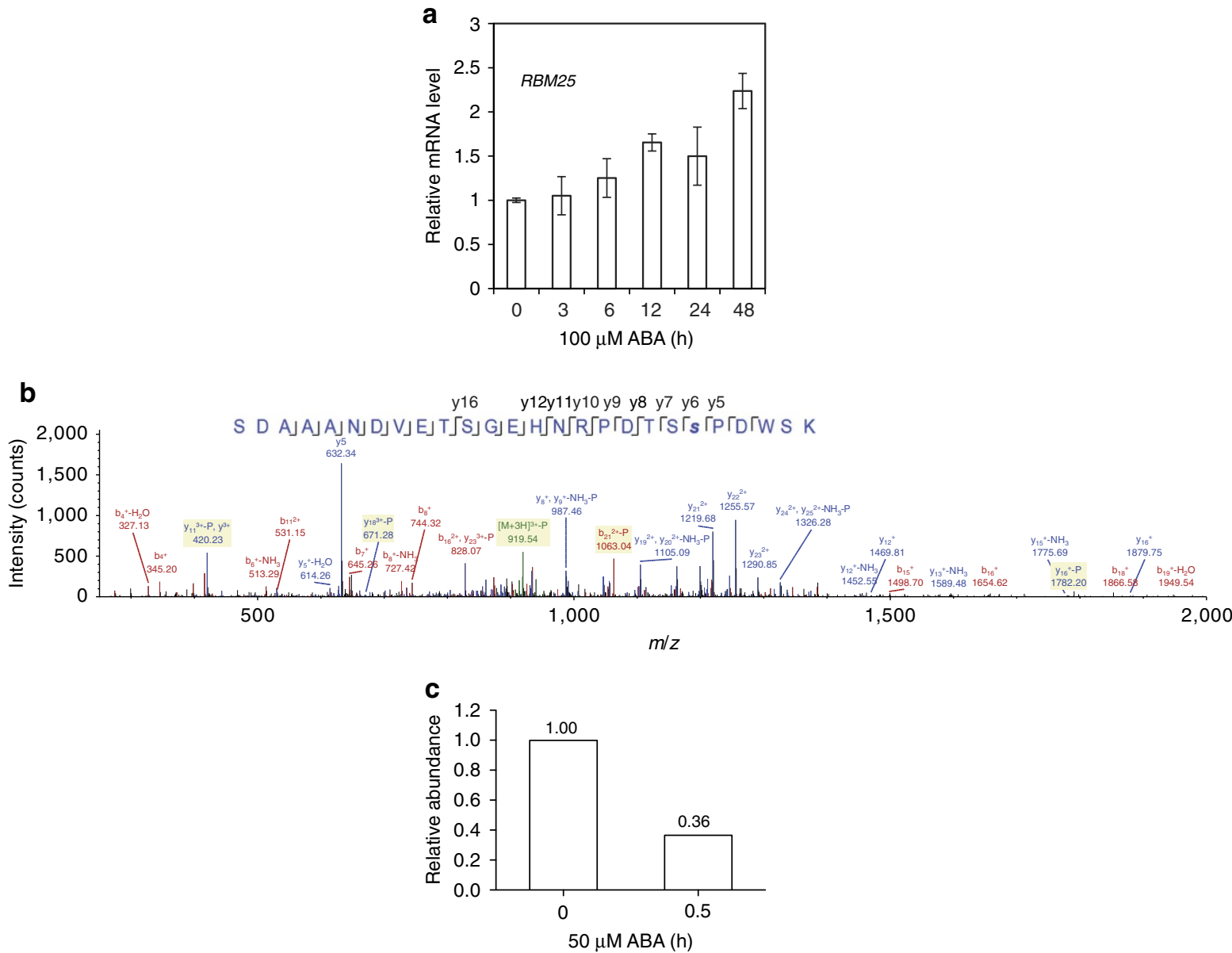

Figure 7 | RBM25 is upregulated by ABA and ABA represses phosphorylation level of RBM25. (a) Relative transcript levels of RBM25 in WT plants as determined by qRT-PCR analysis. Values are mean \pm s.d. $(n=4)$. $(\mathbf{b}, \mathbf{c})$ Quantitative phosphoproteomic profiles of total proteins were performed as described ${ }^{46}$. Proteins were extracted from 12-day-old WT plants subjected to $50 \mu \mathrm{M} \mathrm{ABA}$ treatment for 0 or $0.5 \mathrm{~h}$, and the enrichment of the phosphoproteins and label-free quantification were performed as described ${ }^{46}$. This experiment was repeated three times with similar results. (b) Mass spectrometric spectra of a phosphopeptide (aa 407-432) in RBM25 identified in vivo. (c) Relative abundance of the RBM25 phosphopeptide. 


\section{Discussion}

In our unique genetic screen for regulators of $\mathrm{ABA}$ responses at seedling developmental stage, we identified a splicing factor, RBM25. The rbm25 mutant plants do not exhibit obvious growth and developmental defects under normal growth conditions, and the mutants are not altered in their responses to heat stress, ethylene or auxin. We established the identity of RBM25 through a map-based cloning strategy followed by a gene complementation analysis. Our genetic evidence demonstrated that RBM25 is required for proper $\mathrm{ABA}$ responses in plants by maintaining proper patterns of splicing of gene transcripts. Although splicing is important for all cellular processes involving gene expression, the splicing factor RBM25 is essential for root growth and leaf development in response to ABA but appears not to be required for normal growth and development. The rbm 25 mutants are hypersensitive to $\mathrm{ABA}$ in the growth medium, indicating that RBM25 may be involved in mediating signal transduction events downstream of ABA. Indeed, our RNA-seq analyses revealed that RBM25 controls the splicing of one of the central ABA signalling molecules, HAB1 (Fig. 6). Ectopic expression of HAB1.1 alone can partially suppress the hypersensitive phenotype of $r b m 25-2$ to $\mathrm{ABA}$ in growth medium (Fig. 6). These results indicate that RBM25 is critical for ABA signalling.

Because gene splicing is an essential cellular process, it is difficult to obtain and study null alleles of splicing factor genes due to their lethality. The rbm25-1 and rbm25-2 alleles appear to be null alleles since RBM25 is not expressed in rbm25-1 and the PWI domain, which is presumably important for splicing function, is truncated in rbm25-2 (Fig. 2b,f). To our knowledge, this is the first time null alleles of a conserved splicing factor are found and characterized in plants. The conditional phenotype (that is, under ABA treatment only) of rbm 25 mutants implies that RBM25 is not essential for splicing for all or most plant genes. However, there are some splicing defects in rbm 25 mutant plants even under control conditions, but the splicing defects become more severe under ABA treatment such that growth and developmental phenotypes are manifested by the mutants under ABA treatment. Because rbm 25 mutants lack obvious growth and developmental defects in the absence of ABA or in responses to heat stress and two additional hormones (ethylene and auxin), but have severe phenotypic defects in the presence of ABA (Fig. 1 and Supplementary Fig. 1), we propose that plants may have a particular requirement for splicing in the presence of ABA. Indeed, this splicing factor (RBM25) shows increased expression under ABA treatment, which is consistent with the notion of an exaggerated requirement for splicing in the presence of $\mathrm{ABA}$. On the other hand, we speculate that $\mathrm{ABA}$ signalling also regulates splicing in at least two ways: to increase the level of RBM25 transcript; and regulate the phosphorylation status of RBM25 (Fig. 7; Supplementary Fig. 5) and other splicing factors ${ }^{26}$. The phosphorylation levels of several splicing factors are regulated by $\mathrm{ABA}$ in wild-type plants ${ }^{26}$, which perhaps strengthen splicing so as to help plant cope with $\mathrm{ABA}$ and related environmental stress. In this study, we showed that the phosphorylation level of RBM25 is downregulated by ABA and we propose that reduced phosphorylation of RBM25 might be important for its activity as a splicing factor or its protein stability under ABA treatment although the physiological significance of RBM25 phosphorylation remains to be determined. Because orthologues of RBM25 exist in various plant species (Fig. 2c; Supplementary Fig. 2), the molecular function of RBM25 may be conserved in other plant species including crops.

Our RNA-seq experiments detected many genes whose splicing is controlled by RBM25. We observed that the rbm25-1 mutant plants carry splicing defects in alternative splicing of pre-mRNAs (Supplementary Data 3). Our observation that there are more genes with defective splicing patterns when rbm25-1 mutant was treated with $\mathrm{ABA}$ is consistent with $\mathrm{ABA}$ upregulation of ROA1 (Fig. 7a; Supplementary Fig. 5; Supplementary Data 3). Intron-retained transcripts in rbm25-1 mutant tend to include premature stop codons and therefore produce truncated or even inactive forms of proteins. In addition, many IR events may lead to the generation of nonsense-mediated decay substrates so that the overall level of the transcript would decrease and no translation would take place. Other abnormal, alternatively spliced transcripts in rbm25-1 mutant probably produce nonfunctional proteins, and such splicing events were seldom detected in wild-type plants in our RNA-seq experiments. Several previous studies have shown that mutants with altered sensitivity to $\mathrm{ABA}$ are affected in pre-mRNA splicing under normal developmental conditions ${ }^{49-55}$ or under abiotic stress conditions including drought and salinity ${ }^{56-60}$. In the previous studies, the various mutants were defective in ABA-regulated seed germination and/or stomatal regulation, but not in seedling leaf greening. In addition, the previously published mutants are defective in not only splicing but also other RNA processing events, and the published work did not link the mis-splicing of a particular gene(s) to the mutant phenotypes. In contrast, the rbm 25 mutants are primarily defective in leaf greening in response to $\mathrm{ABA}$, and we have identified the mis-splicing of $H A B 1$ as a critical mis-spliced gene contributing to the mutant phenotype (Fig. 6), although the defective splicing in many other genes may also contribute to the mutant phenotypes of rbm25.

\section{Methods}

Plant materials. T-DNA insertional mutant pool with known T-DNA insertion sites including rbm25-1 (SALK_064472) were obtained from Arabidopsis Biological Resource Center (ABRC; Columbus, OH). rbm25-2 (containing pTCH4:LUC reporter gene ${ }^{29}$; seeds of $p T C H 4: L U C$ line was kindly provided by Dr Janet Braam) was isolated from a T-DNA-mutagenized Arabidopsis $\mathrm{T}_{2}$ population. Seeds were surface sterilized and sown in germination medium $(1 \times$ Murashige-Skoog (MS) salts, $2 \%$ sucrose, $1.2 \%$ agar, pH 5.7). For ABA-sensitivity screening, 5-day-old seedlings were transferred to new MS agar plates supplemented with $5 \mu \mathrm{M}$ ABA and were allowed to grow vertically for an additional 15-20 days. Seeds of hab1-1 (SALK_002104) were obtained from the ABRC. Plants at all developmental stages were grown in a growth chamber at $22 \pm 1{ }^{\circ} \mathrm{C}$ under cool, white light $(\sim 100-$ $\left.120 \mu \mathrm{mol} \mathrm{m}^{-2} \mathrm{~s}^{-1}\right)$ with a long-day photoperiod (16-h light/8-h dark).

Map-based cloning and gene complementation. The rbm25-1 mutant was crossed with the Landsberg erecta accession, and a total of 896 plants homozygous for the rbm25-1 phenotype in response to ABA in MS medium were selected from the $F_{2}$ population. A separate $F_{2}$ population was also generated from a cross between $r b m 25-2$ and Landsberg erecta, and 896 plants homozygous for the rbm25-2 phenotype in response to ABA in MS medium were selected. Simple sequence length polymorphism markers were designed according to the information in the Cereon Arabidopsis Polymorphism Collection and were used to analyse recombination events. Mapping indicated that rbm25-1 and rbm25-2 mutations were located on the BAC clones T30E16 and F8A5. Candidate genes within this region were sequenced from the rbm 25 mutants and compared with those in GenBank to find the rbm25-1 and rbm25-2 mutations. For gene complementation of roal mutants, a 7.4-Kb genomic fragment of At1g60200 including $2.2 \mathrm{~Kb}$ upstream of the translation initiation codon and $0.4 \mathrm{~Kb}$ downstream of the translation initiation codon was amplified by PCR with T13D8 as a template (primers were listed in Supplementary Data 1). The amplified fragment was first cloned via Gateway technology (Invitrogen) into the pENTR1A Dual Selection Vector. The RBM25 gene was then introduced into the pGWB501 vector, resulting in plasmid pGWB501-ROA1. The pGWB501-ROA1 construct was transferred into Agrobacterium tumefaciens (strain GV3101), and rbm25-1 and rbm25-2 plants were transformed by the floral dip method. Transgenic plants were selected on MS agar plates containing $50 \mathrm{mgl}^{-1}$ hygromycin.

Subcellular localization. The $\sim 2-\mathrm{Kb}$ genomic DNA fragment upstream of the start codon of RBM25 (At1g60200) was amplified by PCR with T13D8 as a template using the primer pair At1g60200P-F5 and At1g60200P-R2, resulting in DNA fragment I. The coding region of At1g60200 (from the start codon to the last codon without stop codon or any intron sequences) was amplified by PCR with the primer pairs At1g60200CDS-F2 and At1g60200CDS-R2, resulting in DNA fragment II. The DNA fragments I and II were joined together by PCR amplification using the primer pairs At1g60200P-F5 and At1g60200CDS-R2 with DNA 
fragments of I and II as a template. The resulting DNA fragment III was cloned into a vector pENTR1A initially and subsequently into the binary vector pGWB504, resulting in the plasmid RBM25:RBM25-GFP. The RBM25:RBM25GFP construct was then introduced into Arabidopsis wild-type (Col-0) plants by floral dip transformation with A. tumefaciens strain GV3101. The subcellular localization of RBM25:RBM25-GFP protein in roots of transgenic plants ( $\mathrm{T}_{2}$ generation) was determined with a Leica SP5X confocal microscope (Leica Microsystems).

\section{Construction of the 35S:HAB1.1 and 35S:HAB1.2 plasmids. The CDNA} fragments were amplified by PCR with primer pairs HAB1CD-F1 plus HAB1CDR1 (for HAB1.1) and HAB1CD-F1 plus HAB1CD-R2 (for HAB1.2). The amplified cDNA fragments were initially cloned into the vector pENTR-D/TOPO and subsequently into the binary vector pGWB2 to generate plasmids 35S:HAB1.1 and 35S:HAB1.2. These plasmids were then introduced into Arabidopsis wild-type (Col-0) plants into rbm25-2 plants by floral dip transformation with A. tumefaciens strain GV3101.

RNA-seq data analyses and validation experiments. Fourteen-day-old wildtype and $r b m 25-1$ seedlings grown on MS medium $(1 \times$ MS salts, $2 \%$ sucrose, $0.6 \%$ agar, $\mathrm{pH}$ 5.7) were treated with 0 or $100 \mu \mathrm{M} \mathrm{ABA}$ for $6 \mathrm{~h}$ and were used for total RNA extraction. Total RNA was isolated with the Universal Plant Total RNA Extraction Kit (BioTeke) and treated with TURBO DNA-free Kit (Ambion) to remove any genomic DNA contaminants. mRNA-seq libraries were constructed following the standard Illumina protocols. There are three biological replicates per genotype. Illumina sequencing was performed in the Shanghai Center for Plant Stress Biology with an Illumina HiSeq 2500 System.

For each sample, RNA-seq raw reads were trimmed using Trimmomatic v0.32 and PRINSEQ v0.20.4 (refs 61,62). Trimmomatic was used to remove the potential Illumina adapter contamination and conduct read trimming and clipping the low quality bases. PRINSEQ was subsequently employed to mainly remove lowcomplexity reads. The remained reads were then aligned to $A$. thaliana genome sequence and the reference-annotated genes (TAIR10) using TopHat v2.0.13 programme ${ }^{63}$ with customized parameters specially for our RNA-seq libraries from plant $A$. thaliana (--read-edit-dist 3--read-realign-edit-dist 0--b2-very-sensitive-r 150--mate-std-dev 200-a 6-i 8-I 10000--min-segment-intron 8--max-segmentintron 10000--microexon-search).

According to RNA-seq-mapped reads and the reference-annotated transcripts, transcriptomes were reconstructed for each sample by Cufflinks v2.2.1 (ref. 64). Given variable efficiency of mRNA enrichments and rRNA depletion kits in samples, these transcripts were masked in transcriptome constructions to improve the overall robustness of transcript abundance estimates. To obtain a high confidence of transcriptomes, the novel constructed transcript was filtered out when the abundance was $<20 \%$ (default is $10 \%$ ) of the most abundant isoform for each gene. Then all of the constructed transcriptomes were merged with the reference-annotated transcripts using Cuffmerge ${ }^{64}$ to yield comprehensive reannotated gene transcripts including known and novel annotated transcripts in our RNA-seq samples. Subsequently, significantly differentially expressed genes were predicted by Cuffdiff ${ }^{64}$ between the controls and tested samples, using twofold change and multiple test $P$ value $<0.05$.

Given that IR is the most frequent alternative splicing (AS) type in plants ${ }^{65,66}$ and juncBASE especially improves the reliability of the detection for such AS type $^{67}$, we adopted juncBASE v0.6 to identify AS events within genes for our samples. First, two different databases were constructed, one is based on comprehensive re-annotated gene transcripts (as described above) to identify all internal AS events (not alternative first or last exon events), and the other is derived from just the reference-annotated transcripts to define alternative first and last exons. Then, a series of python scripts from juncBASE were employed to identify exon-exon junctions, exon-intron junctions and AS events, qualify the events and give significantly differentially splicing events between samples. Accordingly, juncBASE-detected AS events ranged from $\sim 15,500$ to $\sim 20,500$, and among the events 358 to 1,464 ones showed significantly differentially AS events $(P$ value $<0.05$ and abs (delta_val) $>5$ ) between pair-wise samples. Moreover, a module from CASH (comprehensive alternative splicing hunting, the renewed version of ASD (alternative splicing detector ${ }^{68}$ ) was used to rescue 19 to 50 differential IR events. Only genes with alternative splicing patterns in all three biological replicates of rbm25-1 mutant plants are listed as differentially alternatively spliced genes.

For RT-PCR and qRT-PCR analyses, $5 \mu$ g of total RNA was used for synthesis of the first-strand cDNA. Each experiment had three to four biological replicates and was repeated at least three times. The comparative cycle threshold (ct) method was applied for calculating gene expression levels, and ACT2 was used as a reference gene.

\section{References}

1. Zhu, J.-K. Salt and drought stress signal transduction in plants. Annu. Rev. Plant Biol. 53, 247-273 (2002).

2. Nambara, E. \& Marion-Poll, A. Abscisic acid biosynthesis and catabolism. Annu. Rev. Plant Biol. 56, 165-185 (2005).
3. Cutler, S. R., Rodriguez, P. L., Finkelstein, R. R. \& Abrams, S. R. Abscisic acid: emergence of a core signaling network. Annu. Rev. Plant Biol. 61, 651-679 (2010).

4. Zeevaart, J. A. D. In: Biochemistry and Molecular Biology of Plant Hormones (eds Hooykaas, P. J. J., Hall, M. A. \& Libbenga, K. R.) 189-207 (Elsevier Science, 1999).

5. Qin, X. Q. \& Zeevaart, J. A. D. The 9-cis-epoxycarotenoid cleavage reaction is the key regulatory step of abscisic acid biosynthesis in water-stressed bean. Proc. Natl Acad. Sci. USA 96, 15354-15361 (1999).

6. Wang, Z. Y., Xiong, L., Li, W., Zhu, J.-K. \& Zhu, J. The plant cuticle is required for osmotic stress regulation of abscisic acid biosynthesis and osmotic stress tolerance in Arabidopsis. Plant Cell 23, 1971-1984 (2011).

7. Ober, E. S. \& Sharp, R. E. Proline accumulation in maize (Zea mays L.) primary roots at low water potentials (I. Requirement for increased levels of abscisic acid). Plant Physiol. 105, 981-987 (1994).

8. Bray, E. A. Classification of genes differentially expressed during water-deficit stress in Arabidopsis thaliana: an analysis using microarray and differential expression data. Ann. Bot. 89, 803-811 (2002).

9. Neill, S. J. \& Burnett, E. C. Regulation of gene expression during water deficit stress. Plant Growth Regul. 29, 23-33 (1999).

10. Fujii, H. et al. In vitro reconstitution of an ABA signaling pathway. Nature 462, 660-664 (2009).

11. Ma, Y. et al. Regulators of PP2C phosphatase activity function as abscisic acid sensors. Science 324, 1064-1068 (2009).

12. Park, S. Y. et al. Abscisic acid inhibits type $2 \mathrm{C}$ protein phosphatases via the PYR/PYL family of START proteins. Science 324, 1068-1071 (2009).

13. Gosti, F. et al. ABI1 protein phosphatase $2 \mathrm{C}$ is a negative regulator of abscisic acid signaling. Plant Cell 11, 1897-1910 (1999).

14. Saez, A. et al. Gain-of-function and loss-of-function phenotypes of the protein phosphatase 2C HAB1 reveal its role as a negative regulator of abscisic acid signalling. Plant J. 37, 354-369 (2004).

15. Umezawa, T. et al. Type $2 \mathrm{C}$ protein phosphatases directly regulate abscisic acid-activated protein kinases in Arabidopsis. Proc. Natl Acad. Sci. USA 106, 17588-17593 (2009).

16. Vlad, F. et al. Protein phosphatases 2C regulate the activation of the Snf1related kinase OST1 by abscisic acid in Arabidopsis. Plant Cell 21, 3170-3184 (2009).

17. Fujii, H. \& Zhu, J.-K. Arabidopsis mutant deficient in 3 abscisic acid-activated protein kinases reveals critical roles in growth, reproduction, and stress. Proc. Natl Acad. Sci. USA 106, 8380-8385 (2009).

18. Fujii, H., Verslues, P. E. \& Zhu, J.-K. Identification of two protein kinases required for abscisic acid regulation of seed germination, root growth, and gene expression in Arabidopsis. Plant Cell 19, 485-494 (2007).

19. Geiger, D. et al. Activity of guard cell anion channel SLAC1 is controlled by drought-stress signaling kinase-phosphatase pair. Proc. Natl Acad. Sci. USA 106, 21425-21430 (2009).

20. Nakashima, K. et al. Three Arabidopsis SnRK2 protein kinases, SRK2D/ SnRK2.2, SRK2E/SnRK2.6/OST1 and SRK2I/SnRK2.3, involved in ABA signaling are essential for the control of seed development and dormancy. Plant Cell Physiol. 50, 1345-1363 (2009).

21. Sato, A. et al. Threonine at position 306 of the KAT1 potassium channel is essential for channel activity and is a target site for ABA-activated SnRK2/ OST1/SnRK2.6 protein kinase. Biochem. J. 424, 439-448 (2009).

22. Geiger, D. et al. Guard cell anion channel SLAC1 is regulated by CDPK protein kinases with distinct $\mathrm{Ca}^{2+}$ affinities. Proc. Natl Acad. Sci. USA 107, 8023-8028 (2010).

23. Lee, S. C., Lan, W., Buchanan, B. B. \& Luan, S. A protein kinase-phosphatase pair interacts with an ion channel to regulate ABA signaling in plant guard cells. Proc. Natl Acad. Sci. USA 106, 21419-21424 (2009).

24. Vahisalu, T. et al. Ozone-triggered rapid stomatal response involves the production of reactive oxygen species, and is controlled by SLAC1 and OST1. Plant J. 62, 442-453 (2010).

25. Umezawa, T. et al. Genetics and phosphoproteomics reveal a protein phosphorylation network in the abscisic acid signaling pathway in Arabidopsis thaliana. Sci. Signal. 6, rs8 (2013).

26. Wang, P. et al. Quantitative phosphoproteomics identifies SnRK2 protein kinase substrates and reveals the effectors of abscisic acid action. Proc. Natl. Acad. Aci. USA 110, 11205-11210 (2013).

27. Munns, R. \& Cramer, G. R. Is coordination of leaf and root growth mediated by abscisic acid? Opinion. Plant Soil 185, 33-49 (1996).

28. Trewavas, A. J. \& Jones, H. G. In Abscisic Acid: Physiology and Biochemistry (eds Davies, W. J. \& Jones, H. G.) 169-188 (Bios Scientific Publishers, 1991).

29. Iliev, E. A. et al. Transcriptional and posttranscriptional regulation of Arabidopsis TCH4 expression by diverse stimuli. Roles of cis regions and brassinosteroids. Plant Physiol. 130, 770-783 (2002).

30. Zhou, A., Ou, A. C., Cho, A., Benz, Jr E. J. \& Huang, S. C. Novel splicing factor RBM25 modulates Bcl-x pre-mRNA 5' splice site selection. Mol. Cell. Biol. 28, 5924-5936 (2008). 
31. Reiter, R. S., Coomber, S. A., Bourett, T. M., Bartley, G. E. \& Scolnik, P. A. Control of leaf and chloroplast development by the Arabidopsis gene pale cress. Plant Cell 6, 1253-1264 (1994).

32. Björklund, S. \& Kim, Y. J. Mediator of transcriptional regulation. Trends Biochem. Sci. 21, 335-337 (1996).

33. Saez, A. et al. Enhancement of abscisic acid sensitivity and reduction of water consumption in Arabidopsis by combined inactivation of the protein phosphatases type 2C ABI1 and HAB1. Plant Physiol. 141, 1389-1399 (2006).

34. Han, S., Tang, R., Anderson, L. K., Woerner, T. E. \& Pei, Z. M. A cell surface receptor mediates extracellular $\mathrm{Ca}(2+)$ sensing in guard cells. Nature 425 , 196-200 (2003).

35. Wang, W.-H. et al. Regulation of the calcium-sensing receptor in both stomatal movement and photosynthetic electron transport is crucial for water use efficiency and drought tolerance in Arabidopsis. J. Exp. Bot. 65, 223-234 (2014).

36. Bartholomew, D. M., Bartley, G. E. \& Scolnik, P. A. Abscisic acid control of rbcS and cab transcription in tomato leaves. Plant Physiol. 96, 291-296 (1991).

37. Weatherwax, S. C., Ong, M. S., Degenhardt, J., Bray, E. A. \& Tobin, E. M. The interaction of light and abscisic acid in the regulation of plant gene expression. Plant Physiol. 111, 363-370 (1996).

38. Allahverdiyeva, Y. et al. Arabidopsis plants devoid of PsbQ and PsbR subunits of the oxygen-evolving complex show altered PSII supercomplex organization and shoRT-term adaptive mechanisms. Plant J. 75, 671-684 (2013).

39. Bossi, F. et al. The Arabidopsis ABA-INSENSITIVE (ABI) 4 factor acts as a central transcription activator of the expression of its own gene, and for the induction of ABI5 and SBE2.2 genes during sugar signaling. Plant J. 59, 359-374 (2009)

40. Zhong, L. et al. Chloroplast small heat shock protein HSP21 interacts with plastid nucleoid protein pTAC5 and is essential for chloroplast development in Arabidopsis under heat stress. Plant Cell 25, 2925-2943 (2013).

41. Kitsios, G., Alexiou, K. G., Bush, M., Shaw, P. \& Doonan, J. H. A cyclindependent protein kinase, $\mathrm{CDKC} 2$, colocalizes with and modulates the distribution of spliceosomal components in Arabidopsis. Plant J. 54, 220-235 (2008).

42. Robert, N., Merlot, S., N'guyen, V., Boisson-Dernier, A. \& Schroeder, J. I. A hypermorphic mutation in the protein phosphatase $2 \mathrm{C} \mathrm{HAB1}$ strongly affects ABA signaling in Arabidopsis. FEBS Lett. 580, 4691-4696 (2006).

43. Rubio, S. et al. Triple loss of function of protein phosphatases type $2 \mathrm{C}$ leads to partial constitutive response to endogenous abscisic acid. Plant Physiol. 150, 1345-1355 (2009).

44. Leonhardt, N. et al. Microarray expression analyses of Arabidopsis guard cells and isolation of a recessive abscisic acid hypersensitive protein phosphatase $2 \mathrm{C}$ mutant. Plant Cell 16, 596-615 (2004)

45. Zeller, G. et al. Stress-induced changes in the Arabidopsis thaliana transcriptome analyzed using whole genome tiling arrays. Plant J. 58, 1068-1082 (2009).

46. Xue, L. et al. Quantitative measurement of phosphoproteome response to osmotic stress in arabidopsis based on Library-Assisted eXtracted Ion Chromatogram (LAXIC). Mol. Cell Proteomics 12, 2354-2369 (2013).

47. Glickman, M. H. \& Ciechanover, A. The ubiquitin-proteasome proteolytic pathway: destruction for the sake of construction. Physiol. Rev. 82, 373-428 (2002).

48. Huang, H. \& Tindall, D. J. Regulation of FOXO protein stability via ubiquitination and proteasome degradation. Biochim. Biophys. Acta 1813, 1961-1964 (2011).

49. Hugouvieux, V., Kwak, J. M. \& Schroeder, J. I. An mRNA cap binding protein, ABH1, modulates early abscisic acid signal transduction in Arabidopsis. Cell 106, 477-487 (2001).

50. Laubinger, S. et al. Dual roles of the nuclear cap-binding complex and SERRATE in pre-mRNA splicing and microRNA processing in Arabidopsis thaliana. Proc. Natl Acad. Sci. USA 105, 8795-8800 (2008).

51. Raczynska, K. D. et al. Involvement of the nuclear cap-binding protein complex in alternative splicing in Arabidopsis thaliana. Nucleic Acids Res. 38, 265-278 (2010).

52. Perea-Resa, C., Hernández-Verdeja, T., López-Cobollo, R., del Mar Castellano, M. \& Salinas, J. LSM proteins provide accurate splicing and decay of selected transcripts to ensure normal Arabidopsis development. Plant Cell 24, 4930-4947 (2012).

53. Golisz, A., Sikorski, P. J., Kruszka, K. \& Kufel, J. Arabidopsis thaliana LSM proteins function in mRNA splicing and degradation. Nucleic Acids Res. 41, 6232-6249 (2013).

54. Zhang, X. N. \& Mount, S. M. Two alternatively spliced isoforms of the Arabidopsis SR45 protein have distinct roles during normal plant development. Plant Physiol. 150, 1450-1458 (2009).
55. Jayaweera, T. et al. Alternative splicing of Arabidopsis IBR5 pre-mRNA generates two IBR5 isoforms with distinct and overlapping functions. PLoS ONE 9, e102301 (2014).

56. Xiong, L. et al. Modulation of abscisic acid signal transduction and biosynthesis by an Sm-like protein in Arabidopsis. Dev. Cell 1, 771-781 (2001).

57. Papp, I., Mur, L. A., Dalmadi, A., Dulai, S. \& Koncz, C. A mutation in the Cap Binding Protein 20 gene confers drought tolerance to Arabidopsis. Plant Mol. Biol. 55, 679-686 (2004).

58. Carvalho, R. F., Carvalho, S. D. \& Duque, P. The plant specific SR45 protein negatively regulates glucose and ABA signaling during early seedling development in Arabidopsis. Plant Physiol. 154, 772-783 (2010).

59. Zhang, Z. et al. Arabidopsis floral initiator SKB1 confers high salt tolerance by regulating transcription and pre-mRNA splicing through altering histone H4R3 and small nuclear ribonucleoprotein LSM4 methylation. Plant Cell 23, 396-411 (2011).

60. Remy, E. et al. A major facilitator superfamily transporter plays a dual role in polar auxin transport and drought stress tolerance in Arabidopsis. Plant Cell 25, 901-926 (2013).

61. Bolger, A., Lohse, M. \& Usadel, B. Trimmomatic: a flexible trimmer for Illumina sequence data. Bioinformatics 30, 2114-2120 (2014).

62. Schmieder, R. \& Edwards, R. Quality control and preprocessing of metagenomic datasets. Bioinformatics 27, 863-864 (2011).

63. Kim, D. et al. TopHat2: accurate alignment of transcriptomes in the presence of insertions, deletions and gene fusions. Genome Biol. 14, R36 (2013).

64. Trapnell, C. et al. Differential analysis of gene regulation at transcript resolution with RNA-seq. Nat. Biotechnol. 31, 46-53 (2013).

65. Syed, N. H., Kalyna, M., Marquez, Y., Barta, A. \& Brown, J. W. S. Alternative splicing in plants-coming of age. Trends Plant Sci. 17, 616-623 (2012).

66. Reddy, A. S. N., Marquez, Y., Kalyna, M. \& Barta, A. Complexity of the Alternative Splicing Landscape in Plants. Plant Cell 25, 3657-3683 (2013).

67. Brooks, A. N. et al. Conservation of an RNA regulatory map between Drosophila and mammals. Genome Res. 21, 193-202 (2011).

68. Zhou, X. et al. Transcriptome analysis of alternative splicing events regulated by SRSF10 reveals position-dependent splicing modulation. Nucleic Acids Res. 42, 4019-4030 (2014).

69. Tamura, K., Stecher, G., Peterson, D., Filipski, A. \& Kumar, S. MEGA6: molecular evolutionary genetics analysis version 6.0. Mol. Biol. Evol. 30, 2725-2729 (2013).

\section{Acknowledgements}

We thank Dr Shingo Nagawa, Youling Zeng, Sanjib Kumar Panda, Blanca E BarreraFigueroa and Viswanathan Chinnusamy for technical assistance. This work was supported by the National Science Foundation Grant IOS0919745 to J.Z., by NIH grant R01GM059138 to J.-K.Z. and by the Chinese Academy of Sciences.

\section{Author contributions}

X.Z., B.Q., F.C., J.Z. and J.-K.Z. designed the research. X.Z. performed the following experiments: isolation and characterization of the roal mutants, mapping and gene complementation, subcellular localization, and isolation of total RNA for RNA-seq experiments and validation of RNA-seq data. J.Z. and Q.G. performed the water loss, heat stress responses, genetic analysis experiments, seed germination and contributed to experiments on responses of seedlings in ABA-containing media. B.Q., X.G., T.A.O and S.L.D. analysed responses of roal mutants to ethylene and auxin. B.Q. performed the multiple sequence alignment and construction of phylogenetic tree of ROA1 and its orthologues. P.W. performed ROA1 phosphorylation profiling experiment. W.W., J.Z., B.Q. and L.Y. analysed the RNA-seq data. X.Z., J.Z. and B.Q. analysed the results and prepared the figures and tables for the article. J.Z., X.Z., and J.-K.Z. wrote the article.

\section{Additional information}

Accession codes: The RNA-seq data have been deposited in NCBI Gene Expression Omnibus (GEO) database under the accession code GSE66737.

Supplementary Information accompanies this paper at http://www.nature.com/ naturecommunications

Competing financial interests: The authors declare no competing financial interests.

Reprints and permission information is available online at http://npg.nature.com/ reprintsandpermissions/

How to cite this article: Zhan, X. et al. An Arabidopsis PWI- and RRM motif-containing protein is critical for pre-mRNA splicing and ABA responses. Nat. Commun. 6:8139 doi: $10.1038 /$ ncomms9139 (2015). 